\title{
Atypical Lipid-Dependent Malassezia Species Isolated from Dogs with Otitis Externa
}

\author{
M. J. CRESPO, M. L. ABARCA, AND F. J. CABAÑES* \\ Departament de Patologia i Producció Animals (Microbiologia), Facultat de Veterinària, \\ Universitat Autònoma de Barcelona, 08193 Bellaterra (Barcelona), Spain
}

Received 28 December 1999/Returned for modification 26 February 2000/Accepted 25 March 2000

\begin{abstract}
During a survey of the occurrence of Malassezia species in the external ear canals of dogs with chronic otitis externa, lipid-dependent Malassezia species were isolated in three dogs. These species were identified as Malassezia furfur and M. obtusa but showed atypical assimilation patterns. To our knowledge, this is the first report of the isolation of lipid-dependent species of the genus Malassezia in association with canine otitis.
\end{abstract}

Species of the genus Malassezia inhabit the skin of humans and many other warm-blooded vertebrates (20). Their most important physiological feature is their lipophilic nature, because they have the property of using lipids as a source of carbon. In fact, with the exception of Malassezia pachydermatis, the remaining species of the genus have an absolute requirement in vitro for supplementation with long-chain fatty acids in the culture medium.

The taxonomy of these lipophilic fungi has always been a matter of controversy. A few years ago, M. furfur (Robin) Baillon 1889, M. pachydermatis (Weidman) Dodge 1935, and M. sympodialis Simmons and Guého 1990 were the only recognized species. Recently, the genus has been revised on the basis of morphology, ultrastructure, physiology, and molecular biology and enlarged to seven species that include the three taxa mentioned above and four new taxa: M. globosa, M. obtusa, $M$. restricta, and M. slooffiae (12).

These yeasts are etiological agents of chronic and superficial skin disorders. In addition, their importance as emergent pathogens in humans is increasing because they have been identified as causative agents of sepsis in immunocompromised patients (1) and neonates receiving parenteral lipid alimentation (23). All lipid-dependent species can be isolated from normal and diseased human skin, and they can become pathogenic under the influence of predisposing factors. They are associated with several diseases, such as pityriasis versicolor, folliculitis, seborrheic dermatitis, and some forms of atopic dermatitis and even systemic infection (11). M. pachydermatis can be isolated from the skin and mucosae of a variety of birds and mammals, including dogs and cats $(9,14)$. Although this species is mainly adapted to nonhuman animals, it has been reported to cause nosocomial systemic infection in humans $(19,24)$. This yeast can be transmitted by human health-care workers from their pet dogs to their neonatal patients (5). It is the most common yeast that contributes to chronic otitis externa in dogs (22).

During a survey of the occurrence of Malassezia species in the external ear canals of dogs with chronic otitis externa, lipid-dependent Malassezia species were isolated in three dogs. To our knowledge, this is the first report of the isolation of

\footnotetext{
* Corresponding author. Mailing address: Departament de Patologia i Producció Animals (Microbiologia), Facultat de Veterinària, Edifici V, Room V0-285, Universitat Autònoma de Barcelona, 08193Bellaterra (Barcelona), Spain. Phone: 349358117 49. Fax: 3493581 20 06. E-mail: javier.cabanes@uab.es.
}

lipid-dependent species of the genus Malassezia in association with canine otitis.

Microbiology. Swabs from the external ear canals of 57 pet dogs (29 male and 28 female) with chronic otitis externa were obtained for microbiologic examination. Each sample was inoculated onto the following media: blood agar, MacConkey agar, Sabouraud glucose agar (SGA), SGA supplemented with olive oil (10 ml/liter), and Leeming's medium (10 $\mathrm{g}$ of peptone, $5 \mathrm{~g}$ of glucose, $0.1 \mathrm{~g}$ of yeast extract, $4 \mathrm{~g}$ of desiccated ox bile, $1 \mathrm{ml}$ of glycerol, $0.5 \mathrm{~g}$ of glycerol monostearate, $0.5 \mathrm{ml}$ of Tween $60,10 \mathrm{ml}$ of whole cow's milk, $12 \mathrm{~g}$ of agar per liter $[\mathrm{pH}$ 6.2]) (17). The last three media were made for mycological examination and contained $0.05 \%$ chloramphenicol and $0.05 \%$ cycloheximide. For cytological examination, specimens were heat fixed and stained with Gram and Diff-Quick stains. Plates of SGA, SGA supplemented with olive oil, and Leeming's medium were incubated at $35^{\circ} \mathrm{C}$ and examined after $3,5,7$, and 14 days. Plates of blood agar and MacConkey agar were incubated at $37^{\circ} \mathrm{C}$ with $5 \% \mathrm{CO}_{2}$ for 3 days. When growth on SGA supplemented with olive oil and/or Leeming's medium was detected, five different colonies were selected from each medium and subcultured on SGA to determine their lipid dependence. $M$. pachydermatis was identified by morphology microscopically and by the ability to grow on SGA. The identification of the lipid-dependent yeasts was based on the ability to use certain polyoxyethylene sorbitan esters (Tweens 20, 40, 60, and 80), as described by Guého et al. (12), and the Tween diffusion test proposed by Guillot et al. (15). The Cremophor EL assimilation test and the splitting of esculin described by Mayser et al. (18) were used as additional tests for the differentiation of the species $M$. furfur, $M$. slooffiae, and M. sympodialis.

Gram and Diff-Quick stains revealed the presence of Malassezia cells in 39 dogs $(68.4 \%)$. Bacteria and Malassezia were seen in 30 of these dogs $(76.9 \%)$, and Malassezia alone was detected in 9 dogs $(23.1 \%)$. More than 10 yeasts per high-power field were observed in 14 of the 39 dogs $(24.6 \%)$, and less than 10 yeasts per high-power field were detected in 25 of them $(43.8 \%)$, but 5 of these last samples were negative for Malassezia in cultures. Bacteria alone were seen in 16 dogs (28.1\%), and for 2 dogs $(3.5 \%)$, the cytological examination was negative. Microbiological cultures were positive for 54 dogs $(94.7 \%)$. Pure cultures for bacteria were obtained for 15 dogs $(26.3 \%)$. Malassezia species were isolated from 39 dogs $(68.4 \%)$, but for 5 of these dogs, typical Malassezia cells were not observed in cytological examinations. Yeasts belonging to other genera were not isolated. M. pachydermatis was the only yeast isolated from $36 \operatorname{dogs}(63.1 \%)$. In 27 of these dogs $(75 \%)$, 


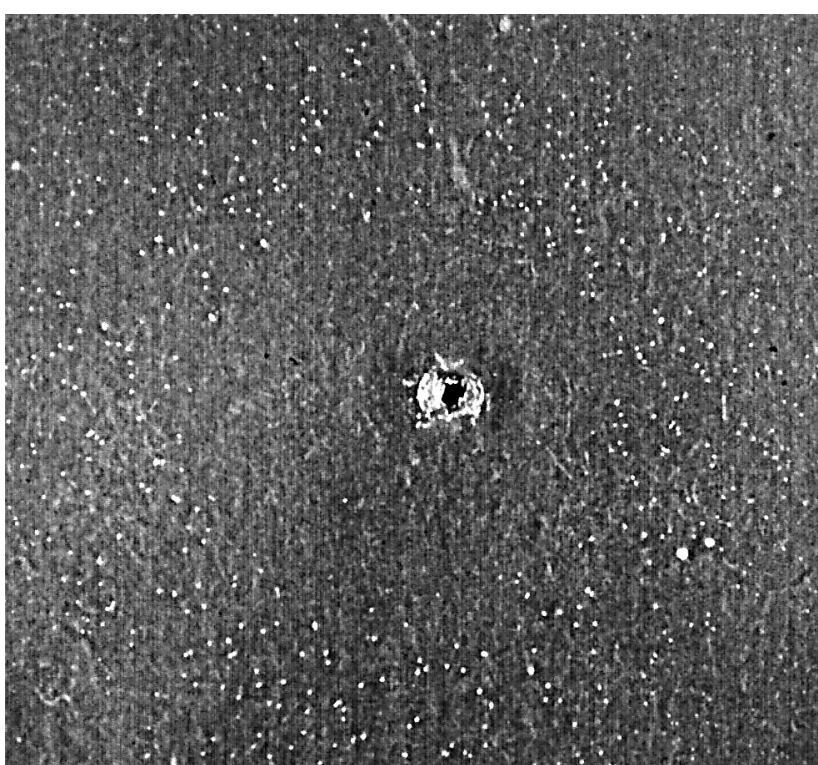

FIG. 1. Cremophor EL assimilation test of an atypical $M$. furfur isolate. Note that an inhibition area around the well is surrounded by a ring of tiny colonies at 10 days of incubation.

the yeast was mixed with bacteria, and only in 9 dogs $(25 \%)$ was the yeast obtained in pure cultures. Lipid-dependent species were obtained in mixed cultures with bacteria from three dogs $(5.3 \%)$. In one of them, the species M. pachydermatis was also isolated.

The lipid-dependent isolates obtained from two dogs formed cream, mat, and smooth colonies with a convex elevation. Their textures were soft, and their average diameters were 3.6 to $5.4 \mathrm{~mm}$ on modified Dixon's agar $(36 \mathrm{~g}$ of malt extract, $6 \mathrm{~g}$ of peptone, $20 \mathrm{~g}$ of desiccated ox bile, $10 \mathrm{ml}$ of Tween 40, $2 \mathrm{ml}$ of glycerol, $2 \mathrm{ml}$ of oleic acid, $12 \mathrm{~g}$ of agar per liter [pH 6.0]) (12) after 7 days of incubation at $32^{\circ} \mathrm{C}$. The cells were cylindrical and oval (1.7 to $2.1 \mu \mathrm{m}$ by 2.9 to $4.3 \mu \mathrm{m}$ ). Buds were formed on a broad base, and short filaments were not observed in any sample. The catalase reaction was positive. The yeasts utilized the four Tweens, grew on glucose-peptone agar with $0.5 \%$ Tweens 40 and $60,0.1 \%$ Tween 80 , and $10 \%$ Tween 20 , and were able to split esculin in 2 to 3 days, as was the type strain $M$. furfur CBS 1878 under the same conditions. These isolates did not grow around the Cremophor EL-containing well at 7 days of incubation. However, an inhibition area measuring 20 to $25 \mathrm{~mm}$ in diameter around the well was surrounded by a ring of tiny colonies after 10 days of incubation (Fig. 1). This pattern clearly differed from the complete disk formed by colonies of the $M$. furfur type strain at 7 days (Fig. $2)$. To confirm that these yeasts really assimilated Cremophor EL, this test was performed again on a single plate, and the same results were obtained at 10 days of incubation. To this end, we confirmed that these lipid-dependent species assimilated Cremophor EL, but not at the high concentration of this compound present near the well. According to these findings, the yeasts had characteristics identical to those of $M$. furfur, with the exception of the growth pattern observed for the Cremophor EL test.

The lipid-dependent yeasts isolated from the third dog formed cream, smooth, and flat colonies with average diameters of 1.0 to $1.4 \mathrm{~mm}$ on modified Dixon's agar after 7 days of incubation at $32^{\circ} \mathrm{C}$. Their textures were sticky, buds were formed on a broad base, and the cells were cylindrical (1.7 to
$2.1 \mu \mathrm{m}$ by 2.4 to $2.8 \mu \mathrm{m}$ ). The catalase reaction was positive, the isolates did not utilize either the four Tweens or Cremophor EL, and the splitting of esculin was positive in 2 to 3 days, as it was for the type strain M. obtusa CBS 7876 under the same conditions. Nevertheless, they were able to grow on glucosepeptone agar with $0.5 \%$ Tweens 40 and 60 , a result which differed from the identification of this species proposed by Guého et al. (12). According to these findings, these lipiddependent species had the same characteristics as the species $M$. obtusa, except for their growth on glucose-peptone agar with $0.5 \%$ Tweens 40 and 60 .

Discussion. The importance of $M$. pachydermatis in dogs has been extensively reported $(13,16)$. This species can play an important role in chronic dermatitis and otitis externa in carnivores, especially in dogs. This yeast has an opportunistic nature, and it may become pathogenic with any alteration in the skin surface microclimate or in host defense. Canine otitis externa and seborrheic dermatitis are frequently associated with large numbers of $M$. pachydermatis (22). The frequency of isolation of this species from the external ear canal is reported to be 15 to $49 \%$ for healthy dogs and may increase up to 50 to $83 \%$ for dogs with otitis externa (10).

Classically, lipid-dependent species were related to human skin only, but it is now known that the skin of different animals also can be colonized by lipid-dependent species in addition to $M$. pachydermatis (14). M. slooffiae seems to represent a large proportion of the cutaneous yeast flora in pigs, and it has been isolated from normal skin in sheep and goats as well (12). The following lipid-dependent species have been isolated from healthy bovines $(8,12)$ : . globosa, $M$. furfur, $M$. slooffiae, $M$. obtusa, and $M$. sympodialis. The presence of lipid-dependent species in carnivores has been recently demonstrated. M. sympodialis (3), M. globosa (4), and M. furfur (6) have been described to colonize the skin and mucosae of healthy cats. $M$. furfur and $M$. sympodialis also have been reported from canine specimens tested by identification techniques such as the assimilation of Cremophor EL, the splitting of esculin, and pigment synthesis (21).

The role of lipid-dependent species in human skin is well

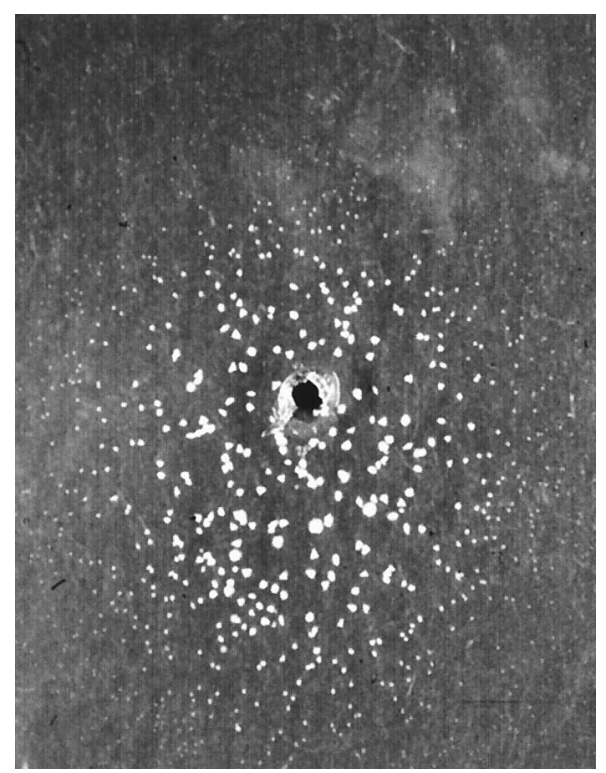

FIG. 2. Cremophor EL assimilation test of the type strain $M$. furfur CBS 1878. Note that a complete disk formed by colonies is observed around the well at 7 days of incubation. 
documented. They are commensal yeasts that may become etiological agents of cutaneous and systemic diseases. However, very little is known about their role in nonhuman animal skin. A skin disorder in goats associated with Malassezia cells morphologically different from $M$. pachydermatis cells was described on the basis of histological techniques, but the isolation in vitro of the casual agent was unsuccessful (2). More recently, lipid-dependent species have been isolated in bovines with otitis externa (M. globosa, M. slooffiae, M. furfur, and M. sympodialis) (8), and two cases of feline otitis externa associated with $M$. sympodialis have been reported from our laboratory (7).

In this study, three atypical lipid-dependent yeasts have been isolated. To our knowledge, this is the first isolation of lipiddependent species in association with canine otitis externa. Lipid-dependent yeasts probably have a pathogenic mechanism similar to that of $M$. pachydermatis in the skin. They are members of the cutaneous flora of some animals, and they might become pathogenic under the same predisposing factors. However, more studies would be required to determine their role in animal skin. For this reason, culture media with lipid sources, such as SGA supplemented with olive oil, Leeming's medium, or modified Dixon's agar, should be used in addition to media without lipid sources. Our finding of lipid-dependent isolates indicates that canine otitis externa can be associated with lipid-dependent Malassezia species in addition to the nonlipid-dependent species $M$. pachydermatis.

On the other hand, the identification system for the lipiddependent species based on the ability to use certain polyoxyethylene sorbitan esters (Tweens 20, 40, 60, and 80), the Cremophor EL assimilation test, and the splitting of esculin was not able to identify our canine lipid-dependent isolates. Some differences in the assimilation patterns were observed on comparison with the type strain patterns. It is likely that the number of lipid-dependent species that do not fit the described type species profiles increases as long as further isolates are obtained from different hosts. In these such situations, complementary tests, including molecular techniques, would be required to differentiate atypical lipid-dependent isolates. On the other hand, the possibility of some variations in assimilation patterns within a species should be considered.

We thank Autonomous University of Barcelona Veterinary Teaching Hospital and various veterinary clinics from the Barcelona region for the samples kindly provided for this investigation.

\section{REFERENCES}

1. Barber, G. R., A. E. Brown, T. E. Kiehn, F. F. Edwards, and D. Armstrong. 1993. Catheter-related Malassezia furfur fungemia in immunocompromised patients. Am. J. Med. 95:365-370.

2. Bliss, E. L. 1984. Tinea versicolor dermatomycosis in the goat. J. Am. Vet. Med. Assoc. 184:1512-1513.
3. Bond, R., R. M. Anthony, M. Dodd, and D. H. Lloyd. 1996. Isolation of Malassezia sympodialis from feline skin. J. Med. Vet. Mycol. 34:145-147.

4. Bond, R., S. A. Howell, P. J. Haywood, and D. H. Lloyd. 1997. Isolation of Malassezia sympodialis and Malassezia globosa from healthy pet cats. Vet. Rec. 141:200-201.

5. Chang, H. J., H. L. Miller, N. Watkins, M. J. Arduino, D. A. Ashford, G. Midgley, S. M. Aguero, R. Pinto-Powell, C. Fordham von Reyn, W. Edwards, M. M. McNeil, and W. R. Jarvis. 1998. An epidemic of Malassezia pachydermatis in an intensive care nursery associated with colonization of health care workers' pet dogs. N. Engl. J. Med. 338:706-711.

6. Crespo, M. J., M. L. Abarca, and F. J. Cabañes. 1999. Isolation of Malassezia furfur from a cat. J. Clin. Microbiol. 37:1573-1574.

7. Crespo, M. J., M. L. Abarca, and F. J. Cabañes. 2000. Otitis externa associated with Malassezia sympodialis in two cats. J. Clin. Microbiol. 38:12631266.

8. Duarte, E. R., M. M. Melo, R. C. Hahn, and J. S. Hamdan. 1999. Prevalence of Malassezia spp. in the ears of asymptomatic cattle and cattle with otitis in Brazil. Med. Mycol. 37:159-162.

9. Dufait, R. 1985. Presence de Malassezia pachydermatis (syn. P. canis) sur les poils et les plumes d'animaux domestiques. Bull. Soc. Fr. Mycol. Med. 14:19-22.

10. Greene, C. E. 1998. Integumentary infections. Otitis externa, p. 549-554. In C. E. Greene (ed.), Infectious diseases of the dog and cat, 2nd ed. The W. B. Saunders Co., Philadelphia, Pa.

11. Guého, E., T. Boekhout, H. R. Ashbee, J. Guillot, A. van Belkum, and J. Faergemann. 1998. The role of Malassezia species in the ecology of human skin and as pathogens. Med. Mycol. 36:220-229.

12. Guého, E., G. Midgley, and J. Guillot. 1996. The genus Malassezia with description of four new species. Antonie Leeuwenhoek 69:337-355.

13. Guillot, J., and R. Bond. 1999. Malassezia pachydermatis: a review. Med. Mycol. 37:295-306.

14. Guillot, J., R. Chermette, and E. Guého. 1994. Prévalence du genre Malassezia chez les mammifères. J. Mycol. Med. 4:72-79.

15. Guillot, J., E. Guého, M. Lesourd, G. Midgley, G. Chévrier, and B. Dupont. 1996. Identification of Malassezia species. A practical approach. J. Mycol. Med. 6:103-110.

16. Guillot, J., E. Guého, M. Mialot, and R. Chermette. 1998. Importance des levures du genre Malassezia en dermatologie vétérinaire. Point Vet. 29:691701.

17. Leeming, J. P., and F. H. Notman. 1987. Improved methods for isolation and enumeration of Malassezia furfur from human skin. J. Clin. Microbiol. 25: 2017-2019.

18. Mayser, P., P. Haze, C. Papavassilis, M. Pickel, K. Gruender, and E. Guého. 1997. Differentiation of Malassezia species: selectivity of Cremophor EL, castor oil and ricinoleic acid for M. furfur. Br. J. Dermatol. 137:208-213.

19. Mickelsen, P. A., M. C. Viano-Paulson, D. A. Stevens, and P. S. Díaz. 1988. Clinical and microbiological features of infection with Malassezia pachydermatis in high-risk infants. J. Infect. Dis. 157:1163-1168.

20. Midgley, G. 1989. The diversity of Pityrosporum (Malassezia) yeasts in vivo and in vitro. Mycopathologia 106:143-153.

21. Raabe, P., P. Mayser, and R. Weiß. 1998. Demonstration of Malassezia furfur and $M$. sympodialis together with $M$. pachydermatis in veterinary specimens. Mycoses 41:493-500.

22. Scott, D. W., W. H. Miller, and C. E. Griffin. 1995. Muller \& Kirk's small animal dermatology, 5th ed. The W. B. Saunders Co., Philadelphia, Pa.

23. Van Belkum, A., T. Boekhout, and R. Bosboom. 1994. Monitoring spread of Malassezia infections in a neonatal intensive care unit by PCR-mediated genetic typing. J. Clin. Microbiol. 32:2528-2532.

24. Welbel, S. F., M. M. McNeil, A. Pramanik, R. Silberman, A. D. Orbele, G. Midgley, S. Crow, and W. R. Jarvis. 1994. Nosocomial Malassezia pachydermatis bloodstream infections in a neonatal intensive care unit. Pediatr. Infect. Dis. J. 13:104-108. 